\title{
Erratum to: Performance Analysis on Two-Way Relay System with Co-Channel Interference
}

\author{
Fei Yang - Meiyu Huang · Sihai Zhang • \\ Wuyang Zhou
}

Published online: 5 April 2013

C) Springer Science+Business Media New York 2013

\section{Erratum to: Wireless Pers Commun DOI 10.1007/s11277-013-1021-3}

In the original publication, there are errors in the authors' affiliation, in Sect. 5 and the Acknowledgments section. The authors' affiliation is correct as shown in this erratum.

The second paragraph in Sect. 5 should read as follows:

The fixation of interferer set does not impact the rationality of our analysis. Outage performance is shown in Fig. 2 with transmission power varying from 0 to $40 \mathrm{~dB}$, to verify the effectiveness of analytic bounds. Lower and upper bounds of outage are separately displayed for $c=4$ and $c=1 / 4$ cases, representing dedicated relay station and user cooperation scenarios, respectively. For the 2TS and 3TS 2-hop schemes, we plot exact bounds as well as asymptotic lines, and they are shown to converge well from medium SINR regime, i.e., about $P_{S}=15 \mathrm{~dB}$ for the large $c$ case, and $20 \mathrm{~dB}$ for the small $c$ case, in which the relay power is relatively low, and the asymptotic regime starts at a high point of power as a result. Diversity gain obtained from MRC in 3TS scheme is also revealed since the outage bounds decline with a greater slope. Only the asymptotic solutions are provided for the 3TS MRC scheme.

The online version of the original article can be found under doi:10.1007/s11277-013-1021-3.

F. Yang $\cdot$ M. Huang $\cdot$ S. Zhang $(\varangle) \cdot$ W. Zhou

Personal Communication Network and Spectrum Spreading Laboratory, Department of Electronic

Engineering and Information Science, University of Science and Technology of China,

Hefei, Anhui, China

e-mail: shzhang@ustc.edu.cn

F. Yang

e-mail: genyang@mail.ustc.edu.cn

M. Huang

e-mail: myhuang@mail.ustc.edu.cn

W. Zhou

e-mail:wyzhou@ustc.edu.cn 
The Acknowledgments section should read as follows:

This work was supported by National Programs for High Technology Research and Development (2012AA01A506, 2012AA011402), the National Major Special Projects in Science and Technology of China under Grant 2013ZX03003011. 